\title{
El modelo de Atención primaria de Servicios Sociales a debate: dilemas y reflexiones profesionales a partir del caso de Navarra
}

\author{
Lucía Martínez Virto ${ }^{1}$; Begoña Pérez Eransus ${ }^{2}$
}

Recibido: 06/02/2017 / Revisado: 17/03/2017 / Aceptado: 02/06/2017

Resumen. El sistema de Servicios Sociales fue concebido en un contexto social muy distinto al actual, caracterizado por el pleno empleo, el elevado potencial integrador de las familias tradicionales y el bajo volumen de personas mayores dependientes. Los cambios sociales de las últimas décadas, tanto en materia de empleo, modelos familiares o aumento de la esperanza de vida como las drásticas consecuencias de la crisis en muchos hogares, han puesto sobre la mesa algunas dificultades profesionales que avivan los debates tradicionales en torno a la indefinición del objeto de su acción, la población diana, su carácter generalista o polivalente, la aparición de nuevos actores o la propia definición de competencias en materia de diseño, gestión y financiación.

El objetivo de este artículo es avanzar en la reflexión sobre cuál debe ser la respuesta del actual modelo de Servicios Sociales a las nuevas necesidades sociales y profesionales. Las cuestiones que se presentan son resultados preliminares de un proceso de reflexión entre profesionales de los Servicios Sociales que ha comenzado a desarrollarse en la Comunidad Foral de Navarra. Si bien el modelo de Atención primaria de Navarra posee especificidades importantes con el de otras comunidades autónomas, las reflexiones iniciadas acometen debates que buscan contribuir a enfrentar cuestiones clave para los servicios sociales en España.

Palabras clave: Servicios Sociales; Atención Primaria; nuevas necesidades; descentralización; especialización.

Sumario: 1. Los Servicios Sociales de Atención primaria en España. 2. Metodología. 3. El modelo de Atención primaria de Servicios Sociales en Navarra. 4. Un cambio de escenario que aviva los debates tradicionales. 5. Dilemas profesionales en la Atención primaria de los Servicios Sociales. 5.1. "Se trabaja en compartimentos estancos". Aportaciones sobre necesidad de mejorar la coordinación para compensar el proceso de descentralización. 5.2. "Es preciso liberar tiempo para la intervención social". Reflexiones sobre los tiempos de trabajo. 5.3 "El tema comunitario queda en tierra de nadie y de todos". Apuntes sobre el impulso del Trabajo Social comunitario. 5.4. "Servicio de autocuidado de profesionales". Propuestas para el acompañamiento profesional. 6. Debates tradicionales ¿los enfrentamos? 7. Apuntes finales: una inevitable reflexión colectiva. 8. Referencias bibliográficas.

Cómo citar: Martínez Virto, L.; Pérez Eransus, B. (2018) El modelo de Atención primaria de Servicios Sociales a debate: dilemas y reflexiones profesionales a partir del caso de Navarra, en Cuad. trab. soc. 31(2), 333-343.

\section{Los Servicios Sociales de Atención primaria en España}

En numerosas ocasiones se ha referido al sistema de servicios sociales en España como el sistema de protección social más joven. De hecho, su implantación como tal surgió a par- tir de la democratización de las instituciones públicas. Hasta entonces, la atención a las situaciones pobreza y necesidad se encontraba vinculada a la beneficencia, donde imperaba un modelo fundamentalmente institucionalizado, fragmentado, y que habitualmente operaba bajo elevados criterios de discrecionalidad

\footnotetext{
Universidad Pública de Navarra, España lucia.martinez@unavarra.es

2 Universidad Pública de Navarra, España

begonia.perez@unavarra.es
} 
(Rodríguez Cabrero, 1996; Subirats, Adelantado, Fernández Prat, Iglesias, Rapoport y San Martín, 2007).

La universalización de los servicios sociales pretendía, en buena medida, superar este modelo de internamiento vinculado a la beneficencia; gestar un sistema de atención más integrado que permitiera abandonar la fragmentación de la red asistencial y acercar los servicios a la ciudadanía. De esta manera, se iniciaba la progresiva implementación de un sistema de servicios sociales cercano al territorio y flexible a las necesidades sociales, pero también destinado atender a las personas con dificultades específicas en el medio abierto y sin necesidad de internamiento (Aguilar, 2013). Si bien este modelo se apoya en un nivel especializado para atender algunas situaciones que requieren una mayor profundidad o especialización, su filosofía se sustenta en lo que se conoce como el Community Care implantado desde los años 1960 en Reino Unido (Sullivan, 2009).

Por lo tanto, el modelo otorgaba a la atención primaria la cercanía a la ciudadanía, la implicación con la comunidad, la prevención de las necesidades sociales y la universalización del sistema a partir tanto de una buena acogida y orientación, como del diseño y la acción sobre el entorno (Aguilar, Corera, Laparra, Liberal y Pérez Eransus, 2001).

Igualmente, el desarrollo del Sistema de Servicios Sociales en España ha estado estrechamente marcado por el proceso de descentralización de las competencias autonómicas. En el artículo 41 de la Constitución Española se señala que: "Los poderes públicos mantendrán un régimen público de Seguridad Social para todos los ciudadanos, que garantice la asistencia y prestaciones sociales suficientes ante situaciones de necesidad, especialmente en caso de desempleo. La asistencia y prestaciones complementarias serán libres". Este hecho fue resultado de fuertes debates relacionados con las características y la naturaleza del sistema, de tal forma que se apostó por un sistema descentralizado que diera respuesta a la cercanía territorial y al desarrollo de las funciones preventivas y comunitarias.

Como resultado de todo ello, el modelo de gestión de los Servicios Sociales avanzó bajo un modelo de doble red, primaria y especializada. El traspaso de estas competencias ha dado lugar a modelos de gestión e implementación con importantes diferencias entre territorios. Hasta qué punto se avanzó en la implantación de este modelo en cada uno de ellos ha sido objeto de numerosas discusiones, e incluso, de procesos de reordenación (Gil, 2010; SIIS, 2015; AEDGSS, 2016). Sin embargo, a pesar de estas diferencias en el desarrollo, todos los territorios comparten cuestiones de fondo pueden dar lugar a dilemas y debates similares.

A lo largo de este artículo se presentan las líneas de reflexión profesional iniciadas en $\mathrm{Na}$ varra como resultado de un proceso profesional y técnico que tiene como objetivo evaluar el modelo de gestión de la Atención primaria en los Servicios Sociales del Ayuntamiento de Pamplona. El texto no ambiciona proponer alternativas ni acometer los debates abiertos, que deben ser enfrentados en colectivo y de manera participada con todos los actores implicados. Sin embargo es motor de este trabajo compartir con la comunidad académica y profesional los primeros avances de un proceso de reflexión que pone de nuevo sobre la mesa cuestiones de base vinculadas al objeto de los servicios sociales. El objetivo en última instancia es animar este debate, compartir algunas cuestiones e iniciar un proceso colectivo de reflexión que permita avanzar en una mayor precisión en torno a la población diana, los modelos de intervención, la coordinación interdepartamental, el debate sobre la especialización o la polivalencia profesional y la relación con otros proveedores privados o del tercer sector.

Para ello, se presenta un texto articulado en cuatro apartados, precedidos por las notas metodológicas. En primer lugar, se analizan las características del modelo de servicios sociales de Atención primaria en Navarra. En segundo lugar, se contextualiza esta reflexión a partir del impacto que los cambios sociales acontecidos han podido tener en la tipología de demanda, recursos disponibles o modelos de intervención de los servicios sociales. En tercer lugar, se presentan los dilemas profesionales identificados en el proceso de reflexión iniciado en Navarra. Finalmente, se apuntan vías de debate y reflexión común en torno al modelo de Atención primaria de servicios sociales en España.

\section{Metodología}

Los resultados de este trabajo son parte de un proceso de evaluación del modelo de gestión de la atención primaria de Servicios Sociales 
iniciado por el Ayuntamiento de Pamplona. La elección del territorio se debe a que, tanto por volumen de población atendida como por el nivel de descentralización de los programas, lo convierten en un análisis de caso pertinente para testar los resultados del modelo derivado de la Ley Foral 15/2006 de 14 de diciembre, de Servicios Sociales.

El proceso de recogida de información de la primera fase del proceso $^{3}$ ha seguido un desarrollo metodológico eminentemente cualitativo. En primer lugar se desarrolló una recogida de información sobre las dificultades y dilemas profesionales que entraña el actual modelo de Atención primaria. Para ello se creó un grupo motor compuesto por 12 profesionales de Trabajo Social del Ayuntamiento de Pamplona. A partir de este grupo se impulsó un proceso de recogida de información que incorporaba debilidades, amenazas, fortalezas y oportunidades del actual modelo. Este análisis DAFO fue trabajado por los/as profesionales de las 12 Unidades de Barrio de Pamplona. En cada uno de estos equipos participaron un total de dos profesionales de Trabajo Social y un educador/a. El tamaño de cada una de las unidades de barrio depende de las necesidades sociales del territorio, por lo que se acordó un criterio homogéneo de participación de equipos mínimos. Por lo tanto, en esta fase de recogida de información participaron en total 36 profesionales.

Posteriormente, se desarrollaron dos grupos focales de profesionales de servicios sociales de las distintas áreas territoriales de Navarra. En cada grupo participaron dos profesionales de cada una de las 6 áreas de servicios sociales. Es decir, un total de 12 participantes por grupo. Si bien la reflexión iniciada tenía como población de estudio el área de Pamplona, se consideró de interés abrir el debate a las experiencias que el modelo estaba teniendo en otras zonas del territorio, fundamentalmente, rurales.

Ambas fases de campo tenían como categorías de análisis la metodología de trabajo de caso, grupal o comunitaria; tiempo de trabajo de gestión, trabajo en equipo o atención direc- ta; ámbito de acción; valoración de las herramientas de intervención y recogida de información; y los dilemas profesionales.

A partir de la información obtenida ${ }^{4}$, se elaboró un documento que fue devuelto a todos los participantes, con el fin de elaborar un diagnóstico previo de consenso. Con ello se constató que, además de las limitaciones y potencialidades identificadas en relación al modelo de gestión, los resultados cristalizaban debates importantes vinculados a la población diana de los servicios sociales, al modelo de atención social y/o a la relación con otros sistemas e instituciones. Las reflexiones presentadas en este texto son fruto de estas dos primeras fases del campo y buscan contribuir, en la medida de lo posible, a enfrentar algunos de estos debates en el marco de la profesión del Trabajo Social en España.

\section{El modelo de Atención primaria de Servicios Sociales en Navarra}

El desarrollo de los servicios sociales en Navarra se remonta a la Ley Foral 14/1983 de Servicios Sociales, una de las normas pioneras en el Estado español. Con carácter general, las primeras leyes autonómicas de Servicios Sociales se sustentaban en principios generalistas que establecían las bases para desarrollar un marco de protección social autonómico, fundamentalmente destinado a paliar las situaciones de pobreza y exclusión social (Arias, Guillén, Pérez y De Lucas, 2004). A esta norma navarra se le sumó, 7 años más tarde, la Ley Foral 9/1990, de 13 de noviembre, sobre el régimen de autorizaciones, infracciones y sanciones en materia de servicios sociales, la cual contribuyó la sentar las bases sobre las que desarrollar los diferentes recursos de intervención en el sistema.

Este primer marco normativo permitió avanzar de manera muy notable en el desarrollo de las políticas sociales en Navarra. Sin embargo, al igual que en el resto de comunidades autónomas, eran normas sustentadas en el asistencialismo, por lo que la ciudadanía no

La primera fase de recogida de información fue entre marzo y junio de 2016. La segunda fase se desarrolló en los meses de septiembre y octubre de ese mismo año.

La primera fase cuenta con soporte documental en papel, ya que cada categoría de análisis se trabajó en grupos simultáneos. Los grupos focales fueron parcialmente transcritos de manera simultánea, debido a la necesidad de vincular las reflexiones compartidas a cada uno de los profesionales participantes. Su perfil y entorno rural de referencia explicaban buena parte de los planteamientos señalados. La explotación de ambos soportes se realizó en base a las categorías presentadas. El análisis del contenido ha tenido un enfoque inductivo. 
había alcanzado todavía el verdadero derecho al sistema (Aguilar, Corera, Laparra, Liberal y Pérez Eransus, 2001). Esta razón, unida a una necesidad de adaptación de los servicios a las necesidades sociales, motivó la Ley Foral 15/2006, norma vigente en la actualidad. La nueva ley fue fruto de un intenso debate y consenso que llevó a reordenar la atención primaria en servicios sociales (idem).

Sin embargo, a pesar del avance de esta norma, tanto en términos de derecho como en reconocimiento a la responsabilidad pública con el desarrollo de la Cartera de Servicios Sociales a través del Decreto Foral 69/2008, uno de los cambios más significativos de la Ley Foral 15/2006 fue, sin duda, el diseño de una nueva estructura de Servicios Sociales. Esta ambicionaba romper, a través de los equipos de Atención primaria, la tradicional separación entre una primera basada en la acogida y la posterior derivación a los servicios sociales especializados.

Tras un proceso de trabajo colectivo y participado se acordó un modelo de Atención primaria más especializado, que incluía acciones de detección de necesidades y prevención, y se encontraba más integrado en el ámbito comunitario. Para ello, se diseñó un modelo de Atención primaria integrado por 4 programas:

- El Programa de Acogida y Orientación Social, dirigido a toda la población, que ofrece intervención social a las personas que presentan demandas ante los servicios sociales.

- El Programa de Promoción de la Autonomía Personal y Atención a las Personas en situación de Dependencia tiene como objetivo posibilitar a las personas en situación de dependencia la permanencia en su medio habitual el máximo tiempo posible.

- El Programa de Incorporación Social en Atención Primaria, cuya finalidad es favorecer la inclusión social de personas en riesgo o en situación de exclusión social en cualquiera de sus ámbitos.

- El Programa de Atención a la Infancia y Familia en Atención Primaria que tiene como objetivo asegurar a los menores de edad un entorno que permita su desarrollo personal, mediante mecanismos de protección, apoyo personal, familiar y social.
Al igual que ocurrió en el germen del sistema estatal, el nuevo modelo de Servicios Sociales de Atención primaria en Navarra se asentaba en la filosofía de integrar el Sistema de Servicios Sociales en la comunidad, y tenía como objetivo fundamental tratar de superar las dificultades que entrañaba el modelo de Atención primaria generalista existente: una red fragmentada, falta de especialización de los profesionales, un modelo de atención dependiente de la demanda, un desigual desarrollo comunitario y poca participación de los actores implicados (Aguilar, 2014).

La implementación de este modelo en Navarra, entre los años 2007 y 2008, coincidió con el impacto de la crisis económica y con las políticas de austeridad. El cambio de escenario y de reglas del juego modificó por completo la dinámica de atención de las unidades de servicios sociales, dando lugar a debates y dilemas profesionales derivados tanto de la realidad coyuntural de los servicios como de la necesidad de acometer algunos debates de calado orientados a definir mejor una estrategia de acción sobre la población diana y la priorización de recursos, entre otras cuestiones.

Además de la crisis, el modelo mostraba algunos aspectos insuficientemente definidos que también contribuyeron a complejizar la implementación de esta nueva estructura. Por ejemplo, la cartera de Servicios Sociales, que tenía como misión dotar de herramientas a los profesionales y garantizar un desarrollo territorial equitativo, mostraba cierto carácter generalista. Por otro lado, no se tuvo en cuenta la posible e inminente privatización de algunos equipos especializados, por lo que el proceso estuvo insuficientemente planificado. Por último, la cartera también recoge vías de financiación a entidades sociales que no están garantizadas y, en la práctica, no acaban de consolidarse como herramientas profesionales. En definitiva, el nuevo modelo, a pesar de contar con elementos muy novedosos y con grandes potencialidades, encontró frenos tanto en el escenario de crisis como en algunas cuestiones de diseño insuficientemente previstas.

\section{Un cambio de escenario que aviva los debates tradicionales}

El sistema de Servicios Sociales se concibió en un contexto social muy distinto al actual. En aquel momento, el pleno empleo masculino y 
la presencia de empleo estable ofrecían cierta estabilidad económica a buena parte de los hogares. Igualmente, el potencial integrador de las familias tradicionales brindaba cuidado y atención de menores y dependientes. Sin embargo, desde hace unos años los servicios sociales se enfrentan a nuevos riesgos sociales (Taylor-Gooby, 2004; Moreno, 2010): la fuerte destrucción de empleo y tendencia a la precarización de las condiciones laborales, un fuerte incremento de la esperanza de vida que ha contribuido a un aumento del volumen de población dependiente, así como, unas transformaciones familiares que conllevan, en ocasiones, disfunciones en la convivencia de los hogares. La interrelación de estas problemáticas económicas, sociales y de convivencia contribuye a incrementar de manera importante la demanda potencial de los servicios sociales de la red primaria y especializada.

De manera paralela a estos cambios, la coyuntura económica ha impulsado una fuerte contención del gasto social. La austeridad en este espacio ha sido creciente y se ha traducido en procesos de deterioro de las políticas sociales que afectan de manera intensa a los servicios sociales (Mestrum, 2014). En este sentido, destaca que en los últimos años se produce una tendencia asistencial progresiva de las prestaciones de ingresos mínimos, se incrementa el nivel de participación de actores privados en la provisión de servicios públicos y se produce un incremento del discurso activador en las políticas sociales (Carter y Witworth, 2016). Todos estos cambios están impactando fuertemente en el conjunto de redes de servicios sociales de toda Europa, dando lugar a un incremento de la demanda de los servicios sociales con un fuerte constreñimiento de la capacidad de protección (European Social Network, 2016).

Sin embargo, en España, estas tendencias de cambio generalizado se han visto intensificadas. En primer lugar, por un fuerte proceso de destrucción de empleo y precarización que da lugar a un creciente número de hogares en desempleo o en pobreza laboral. Por otro lado, por un sistema de protección al desempleo que presenta importantes lagunas para proteger a personas con trayectorias laborales precarias. Por último, debido a un modelo de acceso a la vivienda en propiedad que ha dado lugar a importantes situaciones de endeudamiento y/o procesos de exclusión residencial (Foessa, 2014).
La combinación de estos tres factores caracteriza a una buena parte de la demanda que llega a los servicios sociales en España: pobreza, falta empleo o empleo precario, agotamiento o ausencia de prestaciones y gastos elevados de vivienda. A partir de aquí la presencia o no de menores, de problemas de salud física o mental, y el propio paso del tiempo contribuyen a la complejidad de las situaciones (De la Red, 2014).

El volumen y la naturaleza de estas situaciones trasciende, sin duda, a la capacidad de integración de los servicios sociales, evidenciando la necesidad de impulsar acciones trasversales que impliquen soluciones integrales tanto para nuestro mercado laboral y sistema de garantía de ingresos, como para el modelo de gestión pública de la vivienda (Fantova, 2014). Sin embargo, como se verá a continuación, uno de los resultados más inmediatos que se están produciendo en los servicios es el incremento de dilemas profesionales vinculados al objeto y sujeto potencial de los servicios. Cuestiones también presentes en otros contextos, pero que no resultaban tan cotidianas.

\section{Dilemas profesionales en la Atención primaria de los Servicios Sociales}

El proceso de evaluación del modelo de Atención primaria en Navarra ha avivado debates vinculados a decisiones ya acometidas en los servicios sociales pero que continúan siendo centro de discusión. De nuevo, la descentralización de los servicios, la coordinación entre programas, la necesidad de nuevas estrategias de acción, la población diana y la priorización de recursos o el impulso del Trabajo Social comunitario se cristalizan como elementos clave para la discusión profesional.

A partir de estos debates resulta inevitable no acometer esta discusión. A continuación se presentan las líneas de reflexión abiertas en Navarra. Del mismo modo, cada una de ellas incorpora algunas alternativas y medidas que forman parte de la discusión en el seno de los servicios sociales. A partir de ello, el trabajo busca contribuir a la reflexión colectiva y continuar avanzando en la adecuación de los Servicios Sociales de Atención primaria al nuevo contexto social. 


\section{1. "Se trabaja en compartimentos estan- $\cos ^{5 \%}$. Aportaciones sobre necesidad de me- jorar la coordinación para compensar el proceso de descentralización}

La configuración de un modelo de primera atención con cierto carácter especializado en el ámbito de acogida, incorporación social, familia e infancia y dependencia buscó enfrentar el debate sobre la especialización y/o la polivalencia del perfil de los profesionales desde un primer nivel de atención. Si bien es cierto que el modelo de Navarra ha dado cierta respuesta a esta cuestión, el proceso de descentralización de los servicios y especialización de los programas ha intensificado el riesgo de trabajar de manera estanca sobre todo, cuando la carga de trabajo cotidiana es elevada.

La descentralización de los servicios cuenta con valoraciones muy positivas entre los profesionales. Por un lado, los programas se encuentran más cercanos a la realidad de las familias. Por otro lado, existe un potencial mayor para atender al conjunto de la demanda. Por último, se valora que el contexto es ahora más propicio tanto para el trabajo en equipo dentro de las unidades de servicios como para el desarrollo del trabajo comunitario.

No obstante, la descentralización no ha llegado a desarrollarse por completo, generando en la práctica diaria algunos desajustes relacionados con la identificación de funciones, población diana a atender por cada programa y la ausencia de estrategias de trabajo conjunto que les llevan al "trabajo en compartimentos estancos", según señalan las propias profesionales.

La dinámica diaria y la implementación de un modelo tan novedoso evidenció la necesidad de buscar espacios de coordinación entre programas que permitieran dar respuesta a algunas preguntas como ¿cuál es la diferencia de perfiles entre la población atendida por uno y otro programa?, ¿se producen duplicidades en los diagnósticos y valoraciones de los casos? $\mathrm{Si}$ bien es cierto que las fronteras entre programas como el de autonomía y dependencia han sido menos permeables, el resto de programas comparten casos y derivaciones internas, por lo que encuentran dificultades, en ocasiones, para definir su población diana. Esta evidencia es especialmente intensa con los perfiles que acumulan intensas dificultades en materia de exclusión y/o salud mental.
En este sentido, se observa que, en la práctica, existe cierta disparidad de criterios en relación con el perfil de usuarios que debe ser atendido por cada programa o en los momentos adecuados para su derivación. Esta falta de criterio consensuado también complejiza la relación con otros ámbitos (educación, salud y otros) ya que se establecen contactos con diferentes profesionales dentro de una misma unidad de Servicios Sociales.

Por lo tanto, como respuesta a esta realidad, se identifica cierto consenso profesional en la necesidad de planificar acciones que promuevan el trabajo en equipo, acciones de intervención grupales y el trabajo social comunitario. Se busca con ello construir una "cultura de servicio" que dé lugar a prácticas más integrales. Para ello, se considera indispensable liberar a algunas profesionales para realizar las funciones de coordinación. Esta propuesta, que forma parte de la discusión, es defendida con el fin de impulsar espacios para la supervisión de casos, la coordinación en casos compartidos por los distintos equipos o profesionales, e incluso, para la comunicación de informaciones clave para el trabajo diario (cambios normativos, directrices nuevas, modificaciones de la cartera, etc.).

\section{2. "Es preciso liberar tiempo para la inter- vención social". Reflexiones sobre los tiem- pos de trabajo}

Otra de las cuestiones centrales del debate versa sobre el tiempo destinado a la gestión y la intervención más directa. En general, la elevada carga de trabajo de todos los servicios y el modelo de atención orientado a la demanda impiden el desarrollo de procesos de intervención profesional intensa. Esta realidad se ha visto multiplicada en los últimos años, el aumento de la demanda ha reducido el tiempo de atención por cita, limitando la capacidad de profundizar en algunas cuestiones importantes más allá de la demanda explicitada.

Las listas de espera se viven con mucha preocupación, en algunos servicios superan el mes y medio, por lo que se ha observado un aumento de personas que, tras ese tiempo de espera, no acuden a la cita. Reducir los tiempos de espera para disminuir el "absentismo de citas" ha sido un objetivo de muchos servicios, debido a que es un indicador que evidencia las 
barreras de acceso al sistema; o lo que es lo mismo, el fenómeno conocido en política social como non take up, del que existe cierto consenso internacional en considerarlo un indicador de la ineficiencia del sistema (Eurofound, 2015).

Esta realidad ha incrementado la preocupación de los profesionales por el objeto de su jornada de trabajo. Fruto de este malestar, una profesional del programa de acogida de Atención primaria señala con angustia: "A veces le veo un papel en el bolso de un tema delicado y digo, por favor, que no lo saque que no me da tiempo.".

A partir de estas reflexiones, parece haber cierto consenso en la necesidad de limitar el número de citas para poder avanzar en la recogida de información, los diagnósticos sociales y las valoraciones. Igualmente, se plantea la importancia de flexibilizar la asignación de citas y reservar espacios de gestión interna que permita a las profesionales, con la información reciente, elaborar los informes o derivaciones que el caso requiera.

Por otro lado, se subraya la necesidad de agilizar y simplificar los procedimientos administrativos, implicar a estos profesionales en algunas de estas tareas de gestión, introducir y reforzar metodologías de Trabajo Social con grupos o aprovechar las nuevas tecnologías para conectar con la ciudadanía.

Muchas de estas propuestas cambiarían, de manera significativa, el modelo organizativo dentro de los servicios de Atención primaria. En primer lugar, porque requieren la implicación de todas las figuras profesionales del servicio. Y en segundo lugar, porque modificaría la actual forma de organización, apostando por un modelo de intervención común, centrado en la persona usuaria y que sea más ágil en la gestión de primeras citas y demandas.

\section{3 "El tema comunitario queda en tierra de nadie y de todos". Apuntes sobre el impulso del Trabajo Social comunitario}

La cuestión comunitaria continúa siendo un tema no resuelto. En el actual contexto se intensifica la dificultad histórica de potenciar el desarrollo comunitario en el día a día de la atención primaria. Es cierto que, a pesar del escaso desarrollo de este nivel, se constatan algunas buenas prácticas al respecto. Sin embargo, la situación general en los servicios es de falta de reconocimiento de esta labor, lo cual se traduce en falta de tiempo para este nivel de intervención, escasa formación y ausencia de mecanismos de evaluación.

El trabajo realizado identifica un consenso importante de los profesionales en relación a la necesidad de recuperar e impulsar el trabajo en red de los barrios desde un punto de vista comunitario. Para ello, se sugiere una planificación del trabajo que permita implementar este nivel de intervención, así como, la necesidad de contar con formación continua en esta área. Los resultados alertan de que si el trabajo comunitario no se incluye en la agenda de los profesionales, cuenta con reserva de tiempo y espacios de coordinación, no llegará a consolidarse como un espacio de acción clave de los servicios sociales.

\section{4. "Servicio de autocuidado de profesio- nales". Propuestas para el acompañamiento profesional}

Por último, la complejidad de las nuevas necesidades sociales y los cambios en el perfil de la demanda han alterado la dinámica de acción en los servicios. Por un lado, estos cambios se manifiestan, en algunos casos, como una oportunidad para repensar algunas prácticas profesionales. No obstante, el sentimiento más manifestado por las profesionales es de malestar, sobrecarga y preocupación. En este sentido, los grupos de trabajo señalan cierta preocupación por el bienestar profesional. Como respuesta, se señala la posibilidad de crear un "servicio de autocuidado de profesionales" que redefina las cargas de trabajo, de respuesta a las necesidades formativas pero, fundamentalmente, promueva espacios de reflexión, encuentro y supervisión profesional.

En definitiva, el proceso de evaluación sobre el modelo de Atención primaria iniciado en Pamplona ha puesto sobre la mesa cuestiones intensas que afectan al objeto del sistema, a la cuestión organizativa, al modelo de atención a la población y al bienestar de los profesionales. Estas discusiones abren algunas vías de reflexión en torno a la definición de las funciones, el tipo de intervención y la población diana de cada uno de los niveles de acción. Del mismo modo, ponen en evidencia cuestiones vinculadas a la coordinación entre servicios, programas y otros sistemas de protección social (salud, educación, empleo, vivienda, etc.). Todos estos debates, tradicionalmente vinculados al sistema de servicios sociales desde su 
creación, se avivan en un contexto de cambio social como el actual.

\section{Debates tradicionales ¿los enfrentamos?}

Los resultados presentados evidencian la necesidad de alcanzar algunos puntos de consenso que guíen el futuro de la atención en los servicios sociales. Estas vías de reflexión son cuestiones complejas, pero que deberán acometerse para avanzar en la toma de decisiones sobre la naturaleza de los servicios y los niveles de atención. Asimismo, solo de esta forma se logrará definir el lugar de los servicios sociales dentro de las propias directrices de intervención política.

El primero de los debates impulsados por este proceso versa sobre la cuestión de la universalidad y/o focalización de la atención. En un contexto de elevada demanda es preciso establecer criterios de priorización de la población, tanto en el acceso a los servicios de acogida como en el resto de programas. La universalidad no está reñida con el tratamiento urgente de los problemas más graves, o al menos no lo está en el sistema sanitario. Se pueden establecer criterios de priorización comunes en el acceso a servicios y recursos en función de la intensidad de la exclusión o en función de las posibilidades de prevención del caso, del criterio de "oportunidad" o de su perfil (presencia de menores, maltrato, etc.).

Para evitar que en este proceso tenga mucho peso la discrecionalidad profesional, que bien puede ser positiva o negativa, es necesario revisar los protocolos de intervención con el fin de evitar priorizaciones distintas en función de cada servicio o cada profesional, generando así una situación de desigualdad para la población atendida. En este caso, una tarea útil es reflexionar honestamente sobre la cartera de prestaciones propia mediante preguntas como estas: ¿qué es lo que decimos que hacemos?, ¿qué es lo que realmente hacemos?, ¿a qué tiene derecho la población cuando acude a nuestros servicios?

La segunda cuestión de calado tiene que ver con el tiempo dedicado a la gestión. El contexto actual ha incrementado la demanda de prestaciones en los servicios sociales, suponiendo una buena parte de la jornada laboral de muchos profesionales. Es importante poner de nuevo sobre la mesa la pertinencia de que las profesionales que tramitan prestaciones lle- ven a cabo procesos de intervención social. No son acciones reñidas, de hecho, la gestión de prestaciones genera importante oportunidades para la detección, el diagnóstico y el trabajo en torno a un contrato de inserción. Sin embargo, el incremento de los procesos burocráticos, las limitaciones de actuación en los casos de denegación de ayudas, la escasa disponibilidad de recursos para intervenciones intensas o el aumento de las funciones de control obstaculizan la intervención social en base a la relación de ayuda que, tradicionalmente, ha sustentado la relación usuaria-profesional dentro del Trabajo Social.

En tercer lugar, encontramos el debate sobre el acompañamiento. Existe cierto consenso profesional sobre lo qué es el acompañamiento social y sus implicaciones en el trabajo de gestión de casos. Sin embargo, es preciso avanzar en una valoración real de la capacidad de llevar a cabo esta metodología intensa, el número de casos a los que se les puede garantizar esta técnica intensiva o los niveles de intervención en los que podrá desempeñarse.

En cuarto lugar, cabe destacar la cuestión vinculada a los modelos de inserción social y laboral. La cultura profesional de los servicios sociales se ha forjado en un contexto social de pleno empleo, por lo que la acción profesional tradicional se orientaba al acceso al empleo de la población en situación de pobreza. Igualmente, el potencial integrador que "antaño" tenía el empleo también ha servido a la red de servicios sociales como recurso de inserción para la intervención con otras situaciones de necesidad (salud mental, marginalidad, etc.). El escenario actual de falta de empleo y precarización cuestiona algunas de estas lógicas e inercias profesionales. Esta dificultad se intensifica todavía más cuando las profesionales se enfrentan a la cultura de servicio del sistema de empleo. La tradicional separación entre ambos sistemas ha generado una elevada distancia entre el diseño de las políticas de activación y los perfiles de "empleabilidad" de las personas en procesos de incorporación sociolaboral en los servicios sociales. Aunque sea complejo, es preciso perseguir un escenario de coordinación y colaboración más intenso que permita, desde el diseño, contar con las personas usuarias de los servicios sociales como beneficiarias potenciales de las políticas y programas de empleo.

En quinto lugar se debe acometer el tradicional enfrentamiento entre la especialización 
profesional y la polivalencia de la profesión. Resulta preciso responder al grado de especialización que requieren los casos más complejos. Los resultados presentados evidencian, por un lado, la potencialidad de los perfiles polivalentes, en tanto y cuanto son capaces de dar una primera respuesta integral a la población. No obstante, la complejidad de muchos de los casos que llegan a los servicios requiere de cierta especialización que dé respuesta a cuestiones vinculadas a la inclusión, a la atención a las familias y menores, así como, la relativa a los cuidados y la dependencia. A partir de ello, se podría plantear que la acogida, con mayor capacidad de cobertura, es un nivel adecuado para la detección, elaboración de diagnósticos, intervenciones preventivas o seguimiento de casos. Mientras que la intervención intensa requiere una mayor especialización y disponibilidad para el acompañamiento social.

En sexto lugar se identifica la necesidad de repensar en las herramientas de trabajo y sistemas de información de los servicios sociales, de tal forma que ayuden a agilizar el trabajo, promover la coordinación de casos, realizar el seguimiento, e incluso, tomar decisiones sobre las cargas de trabajo. El objetivo de ello iría también encaminado a trasladar información a otros niveles de intervención o ámbitos de la política social (empleo, salud, vivienda, garantía de ingresos), con el fin de legitimar herramientas como los informes sociales en otros espacios públicos. La falta de reconocimiento de las herramientas profesionales da lugar al riesgo de duplicidad y solapamiento de valoraciones. Del mismo modo, existe consenso profesional en las limitaciones del actual Sistema de Información de Usuarios de Servicios Sociales (SIUSS) para poder evaluar las acciones y el impacto de las intervenciones. Por lo que las nuevas tecnologías son también una buena vía para la mejora de estas herramientas.

Por último, la evaluación ha constatado la necesidad de replantear la organización interna de los servicios. El trabajo en equipo en torno a valoraciones, seguimiento de casos o diseño de acciones compartidas se torna clave para desarrollar intervenciones eficaces que mejoren la relación con la persona usuaria y la acogida por parte del servicio. No obstante, la movilidad laboral que afecta al sector, e incluso, la ausencia de recursos y espacios de encuentro genera cierto riesgo a que cada profesional trabaje con su cartera de usuarios, perdiendo así una esencia básica de la profesión.
Por lo tanto, los resultados presentados a lo largo del texto evidencian la necesidad de discutir sobre estas cuestiones de base en el sistema. No obstante, también el actual contexto cristaliza algunos elementos clave para la gestión de los Servicios Sociales. Entre ellos, el tipo de relación que se mantenga entre red de Atención primaria con otros proveedores mercantiles y no lucrativos debe acometerse. En los últimos años se han multiplicado de manera importante las acciones desarrolladas en el ámbito social por distintos proveedores e incluso, se ha incrementado la emisión de valoraciones sociales para el acceso a recursos externos (bancos de alimentos, kits escolares, etc.). Este hecho hace pertinente decidir cuál va a ser la relación con estos proveedores externos y qué nivel de implicación tendrán los servicios sociales públicos en el desarrollo o implementación de estos recursos.

La mayoría de los debates presentados en este punto son de largo recorrido en el sector por lo que su superación, de partida, parece ser compleja. Sin embargo, la constatación de estos dilemas hace necesaria la creación de un espacio de acuerdo y la toma de decisiones que forjen criterios comunes dentro de la profesión.

\section{Apuntes finales: una inevitable reflexión colectiva}

Los resultados encontrados en Navarra en la primera fase de la evaluación del modelo de Atención primaria evidencian la necesidad de revisar la adecuación del sistema a las nuevas necesidades sociales que se presentan de manera cotidiana en los servicios. El contexto social es distinto del de hace unas décadas, y en la actualidad del de otros países o entornos. Por lo que no resulta útil, para esta cuestión, importar "buenas prácticas" de programas, metodologías profesionales o prestaciones que en otros escenarios han dado buenos resultados. Por ello, inevitablemente, nos toca decidir, en base a los criterios y experiencias de los profesionales, cómo mejorar la labor de la red primaria de servicios sociales a partir del contexto y los recursos disponibles.

En este sentido, podemos diferenciar distintos ámbitos de reflexión que acometer. Entre ellos destacan: la cuestión de la descentralización de servicios; la coordinación entre sistemas; el tiempo destinado a la intervención; 
y la acción en el espacio comunitario. A la luz de estos debates, habrá que enfrentar, inevitablemente, algunas cuestiones de fondo que, aunque no se espera que se superen, requerirán afrontar un mínimo acuerdo. Entre estos dilemas tradicionales destacan: la cuestión entre la universalidad y focalización de la atención; el debate sobre la gestión de prestaciones; el tiempo para el acompañamiento social o el trabajo interdisciplinar, entre otros. Por último, también harán transversal la discusión cuestiones fundamentales, como la presencia de diversos proveedores de servicios sociales (mercantiles y no lucrativos).

Parece oportuno, que cualquier reflexión en torno a las redes de Atención primaria sea coherente con la situación actual y tenga en cuenta el volumen y las características de la demanda, así como, sus posibilidades de intervención e impacto en las problemáticas exis- tentes (pobreza, exclusión, falta de empleo). Por ello, de manera paralela a la discusión, es importante no perder de vista la evolución de las necesidades sociales.

Este proceso complejo de reflexión y toma de decisiones solo puede ser exitoso si se lleva a cabo con la implicación y participación de todas las personas profesionales del ámbito de los Servicios Sociales. Iniciar un proceso abierto y colectivo de reflexión es una buena manera de encarar esta tarea. Tanto las vías tradicionales de debate académico y profesional en torno a revistas especializadas, colegios profesionales o a congresos, como las nuevas oportunidades tecnológicas a través de foros de discusión virtuales o blogs, son espacios que pueden animar a compartir reflexiones que avancen en estas cuestiones. Sea este trabajo también, una pequeña contribución a ello.

\section{Referencias bibliográficas}

Asociación Estatal de Directoras y Gerentes en Servicios Sociales. AEDGSS. (2016). El futuro de los servicios sociales básicos a debate. (XXIII Congreso Anual. Madrid, 11-12 de noviembre de 2016).

Aguilar, M., Corera, C., Laparra, M., Liberal, B. y Pérez Eransus, B. (2001). La atención social en la comunidad. En: Documento Base para un plan de atención comunitaria de Servicios Sociales. [On line]. Disponible en: http://www.navarra.es/home_es/Temas/Asuntos+sociales/Publicaciones/AtencionPrimaria

Aguilar, M. (2013). Los servicios sociales en la tormenta. Documentación Social, 166, 145-167.

Aguilar, M. (2014). Apuntes para un replanteamiento de los Servicios Sociales en España. En: Foessa (ed.), VII Informe sobre exclusión y desarrollo social de España (pp. 338-363). Madrid: Fundación Foessa.

Arias, A., Guillén, M.E., Pérez, D. y De Lucas, F. (2004). La definición de los servicios sociales en las leyes de servicios sociales de "primera" y "segunda generación" en España. Portularia: Revista de Trabajo Social, 4, 507-518.

Eurofound. (2015). Access to social benefits: Reducing non-take-up. Luxemburgo: Publications Office of the European Union. DOI:10.2806/7077. [On line]. Disponible en: http://www.eurofound.europa.eu/ sites/default/files/ef_publication/field_ef_document/ef1536en.pdf

Carter E. y Witworth, A. (2016). Work activation regimes and well-being of unemployed people: rethoric, risk and reality of quasi marketization in the UK programme. Social Policy \& Administration Journal, Doi:10.1111/spol.12206.

De la Red, N. (2014). Trabajo social en tiempos de crisis. En: E. Pastor y M.A. Martínez-Román (coords.), Trabajo Social en el siglo XXI, Una perspectiva internacional comparada (pp. 97-107). Madrid: Editorial Grupo 5.

European Social Network. (2016). Connecting local with local communities. Brighton: ESN. [On line]. Recuperado de: http://www.esn-eu.org/publications/index.html

Fantova, F. (2014). Identidad y estrategia de los servicios sociales. Documentación social, 175, 105-132.

Foessa. (2014). VII Informe sobre exclusión y desarrollo social de España. Madrid: Fundación Foessa. . [On line]. Recuperado de: http://www.foessa2014.es/informe/index.php

Gil, M. (2010). Repensando los Servicios Sociales Municipales. Oportunidades de cambio. Revista Trabajo social hoy, 59, 119-122.

Mestrum, F. (2014). Las políticas Sociales desde una Perspectiva Mundial. Documentación social, 175, 15-34. 
Moreno, L. (2010). Reformas de las Políticas de bienestar: Contexto y nuevos Riesgos sociales. Documento de trabajo $\mathrm{n}^{\circ}$ 19. CCHS-CSIC: Instituto de Políticas y Bienes Públicos (IPP). [On line]. Recuperado de: www.ipp.csic.es/RePec/ipp/.../CSIC-IPP-DT-2010-19_Moreno.pdf

Navarra. (2006). Ley Foral 15/2006 de Servicios Sociales. [On line]. Disponible en: http://www.lexnavarra. navarra.es/detalle.asp? $\mathrm{r}=10855$

Navarra. (2008). Decreto Foral 69/2008 de cartera de Servicios Sociales. [On line]. Disponible en: http:// www.lexnavarra.navarra.es/detalle.asp? $\mathrm{r}=29663$

Rodríguez Cabrero, G (1996). Los Servicios Sociales en España: implantación, generación de empleo y evaluación de su efectividad. En: AA.VV., Las políticas redistributivas (pp. 361-426). Madrid: Fundación Argentaria-Vinor.

SIIS Centro de Documentación y Estudio. (2015). Reordenación de la red de recursos y programas para la inclusión social de Gipuzkoa. Revista Zerbitzuan, 59, 5-38. Doi. http://dx.doi.org/10.5569/11347147.59 .01

Sullivan, M.P. (2009). Social Workers in Community Care Practice: Ideologies and Interactions with Older People. British Journal of Social Work, 39 (7), 1306-1325. Doi: 10.1093/bjsw/bcn059.

Subirats, J., Adelantado, J., Fernández Prat, M., Iglesias, M., Rapoport, A. y San Martín, J. (2007). Los Servicios Sociales de atención primaria ante el cambio social. Madrid: Ministerio de Trabajo y Asuntos Sociales.

Taylor-Gooby, P. (2004). New Risks, New Welfare: The Transformation of the European Welfare. Oxford: Oxford University Press. 